\title{
Skin Diseases Detection Models using Image Processing: A Survey
}

\author{
Nisha Yadav \\ Department of Computer Science \\ Engineering \& Technology \\ Amity University, Haryana
}

\author{
Virender Kumar Narang \\ Director, Amity School of \\ Engineering \& Technology \\ Amity University, Haryana
}

\author{
Utpal shrivastava \\ Assistant Professor \\ Department of Computer Science \\ Engineering \& Technology \\ Amity University, Haryana
}

\begin{abstract}
Now a days, skin diseases are mostly found in animals, humans and plants. A skin disease is a particular kind of illness caused by bacteria or an infection. These diseases like alopecia, ringworm, yeast infection, brown spot, allergies, eczema etc. have various dangerous effects on the skin and keep on spreading over time. It becomes important to identify these diseases at their initial stage to control it from spreading. These diseases are identified by using many technologies such as image processing, data mining, artificial neural network (ANN) etc. Recently, image processing has played a major role in this area of research and has widely used for the detection of skin diseases. Techniques like filtering, segmentation, feature extraction, image pre-processing and edge detection etc. are part of image processing and are used to identify the part affected by disease, the form of affected area, its affected area color etc. This paper presents a survey of various skin disease diagnosis systems using image processing techniques in recent times. A comprehensive study of a number of skin disease diagnosis systems are done in this paper, with different methodologies and their performances.
\end{abstract}

\section{Keywords}

Image processing, skin diseases, ANN, segmentation, image pre-processing, edge detection, filtering.

\section{INTRODUCTION}

The biggest organ of the body is human skin. Its weight lies between six and nine pounds and surface area is about two square yards. Inner part of body is separated by skin from the outer environment. It provides protection against fungal infection, bacteria, allergy, viruses and controls temperature of body. Situations that frustrate, change texture of the skin, or damage the skin can produce symptoms like swelling, burning, redness and itching. Allergies, irritants, genetic structure, and particular diseases and immune system related problems can produce dermatitis, hives, and other skin problems. Many of the skin diseases, such as acne, alopecia, ringworm, eczema also affect your look. Skin can also produce many types of cancers. Image processing is used to detect these diseases by using various methods like segmentation, filtering, feature extraction etc. To get an improved image or to get meaningful information from an image, it is necessary to convert an image into digital form and then perform functions onto that image. It is a part of signal processing. The input is an image and it may be a video, a photograph and output is also another image having same characteristics as input image. Mostly Image Processing models take input samples as 2-D signals and after that they apply fixed signal processing methods to them. It is widely used technology now days and it has various applications in the area of business. It is a new research area within engineering and computer science too. The range of skin diseases is very wide. As you know, approximately eight million people in the UK currently suffered from skin disease. Skin disease doesn't just damage the skin. It can have a large impact on human's daily life [8], destroy confidence of a person, stop their movement, and turn to depression. The worst situation is that, it can even kill. It's a serious issue that needs to be controlled, so it is necessary to take skin diseases very seriously and identify it at an early stage and prevent it from spreading. Detection of a disease depends upon many factors like which parameters are considered for disease detection. Firstly, take an image, apply filters to remove noise from the image, segment the image to extract meaningful information, feature extraction is done on the basis of input parameters and then classify the diseases by using appropriate classifier.

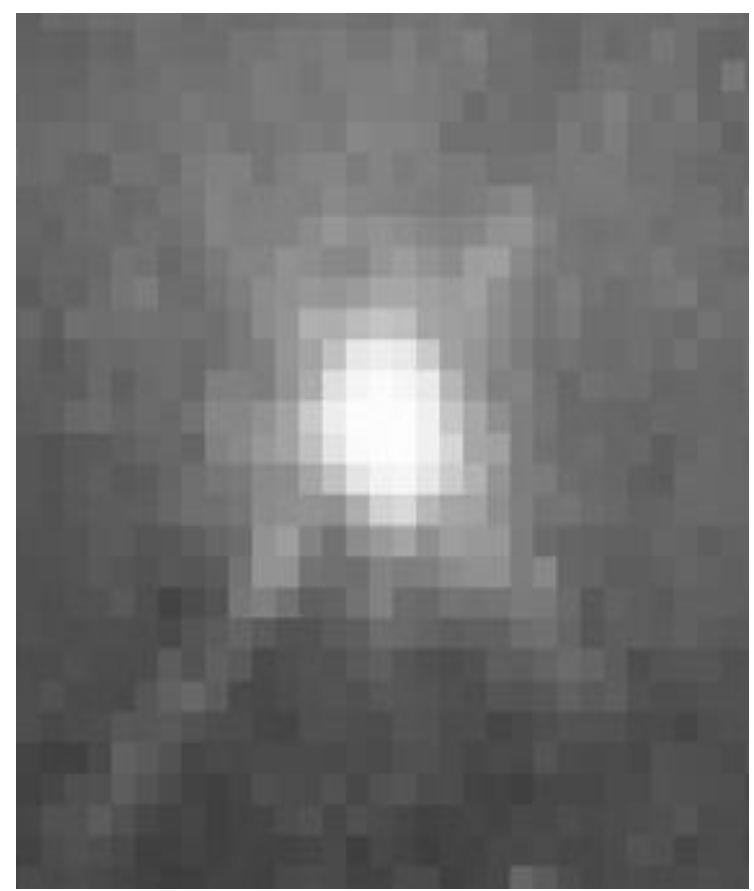

Fig.1.1: An image - collection of pixels arranged in columns and rows.

\section{LITERATURE SURVEY}

\subsection{Skin diseases detection models for plants $[5,19,21]$}

A skin disease in the plant has large economical and production loss. Now a days, there are various diseases [1] that exist on the plant leaves. To increase production rate and quality level it becomes necessary to control these harmful diseases .For this, there is a need to detect a specific disease. Damages in crops occur due to various types of skin diseases. Damage due to insects, fungi's, bacteria's is major diseases. 
Skin diseases in plants mainly exist on leaves, stems. Insecticides are not efficient due to their harmful effects for the bird breeds. It is also dangerous for the animal food process. The main aim for biologist is to calculate the harm of diseases on plant leaves, stems that is occurs due to diseases, by estimating the affected area in plants. In many cases pests or diseases are exist on the leaves, stems of the plant.

\subsubsection{Detection of Leaf Diseases by Image Processing [1]}

In India, mostly peoples are farmers. They have large dependency on the agriculture. They want good quality products. The very important agricultural products from customer point of view are fruits and vegetables. Product quality depends on soil quality, fertilizers and seeds. One major thing which effects production is diseases. To get more profit, it is important to prevent these diseases (Powdery Mildew, Downy Mildew, Black Rot etc.). For this, it is mandatory to identify and control these harmful diseases at their early stage prior it will hinder the basic functionalities of plant internal processes like Photosynthesis, transpiration, pollination, fertilization [34], germination etc. The main reasons for these diseases are pathogens like fungi, bacteria, viruses, and environmental conditions. Various techniques related to image processing can be used to recognize and classify disease in plants.

Algorithm:

1) Image capturing i.e. RGB image.

2) Convert the RGB image into color space i.e. HSV/Lab.

3) Segment the image to get useful information.

4) Feature extraction.

5) Training neural network.

This is a useful model for detection of diseases with some computational effort.

\subsubsection{Guidelines for Identification and Management of Plant Disease Problems: Part II. Diagnosing Plant Diseases Caused by}

Fungi, Bacteria and Viruses [2]

In this module, the main focus is on identifying plant diseases occurring due to pathogenic microorganisms, mainly fungus, bacteria, and viruses. The microscopic "worms" named nematodes create plant related skin problems. The author gives detailed description about these pathogens. In this paper, author discusses about the symptoms and signs of pathogens, Preliminary Diagnostic Equipment, Disease Diagnostic Information and Submission of Samples. The scale of plant diseases that are created due to multi-celled microorganisms called fungi is about $85 \%$. These diseases may be seen with naked eyes during their life cycles stages. Some particular fungi that spend their lifetime inside the soil may get transferred from one plant to other and it will grow with their roots. Bacteria are also called one-celled microorganisms and have small size so you need a powerful light microscope to view them. Bacteria depend upon outside agents for transfer from one plant to other. The smallest pathogens are known as viruses and cannot be seen with naked eyes. You need an electron microscope to view them. They are created by using genetic material i.e. RNA or DNA, which have a layout of protein layer. These Viruses are transferred from disease affected plants to healthy plants by insects. Another way of spreading is mites, nematodes, fungi. Even humans can also be a way of spreading.

\subsubsection{Applying image processing technique to detect plant diseases [3]}

At every place, where you live as well as places without us plants exists. As you know, diseases of the plants are predictable; disease identification plays an important job in the scope of Agriculture. Plant diseases reduce quantity and degrade products quality. In this paper, pomegranate diseases are detected by using image processing. The main problems are diseases and insect pests that damage pomegranate farming. These require supervised treatment and scheduling to secure the crops from heavy losses. The proposed work defines a methodology for plant diseases detection at their early stages and detects them with accuracy, using image processing techniques and artificial neural network (ANN). The work starts with image acquisition. Gabor filter is applied for noise removal and segmentation purpose. Then from the result of segmentation texture based and color based features are extracted and then training of artificial neural network (ANN) is done by selecting those feature values that could help to separate the affected and unaffected images of plants. In this paper, the main focus is on three types of diseases which have large impact on pomegranate crop i.e. Alterneria, Bacterial blight, Anthracnose. Experimental results showed that ANN classify these diseases by using selected feature vector with an accuracy of $91 \%$.In this approach, the neural network is successfully trained with 140 images from which 8 images are alterneria, 26 images are BBD and 89 images are Anthracnose which is selected for training and testing purpose.

\subsubsection{Artificial Neural Network Based Plant Leaf Disease Detection [4]}

As you know, in India wherein approximately seventy percent people depends upon agriculture. Farming in India had a large scope of variety to select particular Fruits and Vegetable crops according to season. In case of plants, the disease is described as any abnormal condition which affects usual physiological processing of plants and creating particular symptoms. Disease is created due to pathogen which creates any agent causing disease. Mostly pests or diseases are found on the plant leaves and stems. So identification of affected plant leaves and stems and estimating the percentage of the pest or disease incidence, symptoms of the pest or disease attack plays a major role in successful farming of crop. It is measured that approximately several billion dollars of loss occurs due to diseases. A dataset of various leaves diseases is prepared for study. For further processing, Artificial Neural Network is used.

\section{Algorithm:}

1. RGB image acquisition.

2. Convert the RGB image to the space specified in the color transformation structure.

3. Apply K-means clustering.

4. Masking green-pixels.

5. Remove the masked cells inside the boundaries of the infected clusters.

6. Convert the infected (cluster / clusters) from RGB to HSI Translation.

7. SGDM Matrix Generation for $\mathrm{H}$ and $\mathrm{S}$.

8. Calling the GLCM function to calculate the features.

9. Texture Statistics Computation.

10. Configuring Neural Networks for Recognition.

11. Comparing two algorithms used for recognition.

The present paper gives the detailed review and description about image processing techniques for various types of plants 
that have been used for detecting plant diseases. The key techniques for the recognition of plant diseases are: BPNN, SVM, K-means clustering, and SGDM. These techniques are mainly used to identify the healthy and unhealthy plants leaves. It shows that this technique has a good ability to detect plant leaf diseases.

\subsubsection{Grading and Classification of Anthracnose Fungal Disease of Fruits based on Statistical Texture Features [5]}

In this paper, those lesion areas which are damaged by disease anthracnose are separated by using segmentation techniques, scaling on the basis of percentage of damaged area and to classify unaffected and anthracnose affected part of fruits, artificial neural network is used. In this paper, mainly three varieties of fruit i.e. mango, grape and pomegranate are used for study. The proposed methodology contains two phases: firstly, segmentation techniques like thresholding, region growing, $\mathrm{K}$-means clustering and watershed are applied for separating damaged lesion areas and unaffected area. Then scaling of the damaged areas is done by estimating the percentage of affected area. In the second phase, Run length Matrix is used to extract texture based features. Classification is done by using ANN classifier on the basis of these extracted features. In this paper, a dataset is prepared of 600 fruits' images. The classification accuracies for unaffected and affected anthracnose fruit types are $84.65 \%$ and $76.6 \%$ respectively. The highest recognition and classification accuracy is measured for normal mango i.e. $87 \%$ and the lowest of $71.5 \%$ are measured with an anthracnose affected a pomegranate. The average accuracy of $84.65 \%$ for normal type and $76.6 \%$ for anthracnose affected type. This work contains importance to real world classification of fruits' disease.

\subsubsection{Recent Studies of Image and Soft Computing \\ Techniques for Plant Disease Recognition and Classification [6]}

The plant diseases are a normal part of nature but can cause significant economic, social and ecologic loss globally. It's difficult to observe continuously plant health and identification of diseases. This paper gives a survey of recent studies on the area of plant disease recognition and classification from digital images using image processing and soft computing techniques. The main goal of this paper is to focus on the plant diseases identification field and classification only. The paper is excluding the disease severity quantification. Although the paper, consider the images of symptoms presents on plant leaves and stems only for limiting the survey. The algorithm starts with image capturing of infected and non-infected plants; perform image preprocessing, differentiate disease affected region from an unaffected region using segmentation, extract features from results of segmentation for recognition and classification. This survey is expected to be useful for researchers from plant pathology and pattern recognition field. The paper presents an overview of technical concepts used in the methods existing in the literature survey related to the goal of the paper.

\subsubsection{An Image Segmentation comparison approach for Lesion Detection and Area calculation in Mangoes [7]}

In food industry, external quality of fruits has a major role which is evaluated by their color, texture and visual lesions. Manual checking is time consuming, costly and non consistent, therefore machine visions approach is used for lesion detection. In this paper, different segmentation techniques are adopted for detection of lesion areas in mango. A cost effective, consistent and accurate detection of lesion area is possible using machine vision. In image processing, various segmentation algorithms are being tested and a best suitable algorithm is selected to identify and to calculate the lesion areas in mango fruit. The proposed system is implemented using defected mangoes. A dataset is prepared about 500 image samples. About 100 samples of mangoes examined and about 30 lesion mangoes were selected for the segmentation process. The proposed system involves; image acquisition, image Pre-processing, segmentation, lesion detection and area calculation. The quality of fruits can be examined by color, appearance, flavor and texture etc. This method has been successfully implemented for lesion detection in mangoes.

\subsection{Skin diseases detection models for humans $[13,14,15]$ :}

2.2.1Expert System for Diagnosis of Skin Diseases [8]

Skin diseases are frequent diseases to every person and various types of infections are becoming very frequent. You know that all of these diseases are very harmful, especially if not controlled at an early stage. Skin diseases not only damage the skin. It can have a large effect on a person's daily life, destroy confidence of a person, hang their movement, and turn to depression. Sometimes, many people try to treat these allergies by using their own therapy. However, if these methods are not appropriate for that type of skin disease then it would make it more harmful. Skin diseases can easily transfer from human to human so there is a need to control it their initial stage to prevent it from spreading. This paper presents an implementation of a skin diseases diagnosis system which helps user to detect human skin diseases and provides medical treatments timely. For this purpose, user will have to upload a disease affected skin image to our system and give answers to the questions which are asked to user according to the symptoms of the skin. These symptoms are used to identify the disease and provide a medical treatment. This system works on technologies like image processing and data mining for skin diseases detection. So the whole project is divided in to below major parts,

- Image preprocessing, segmentation and feature extraction.

- Classification model and skin disease predication.

- Medical treatment suggestions or advice.

The image of skin disease is taken and various preprocessing techniques are applied onto that image for noise removal and image enhancement. This image is segmented by using a segmentation technique i.e. thresholding segmentation. At last, data mining techniques are used to identify the skin disease and to provide recommendations to users. This expert system pertain disease recognition accuracy of $85 \%$ for Eczema, $95 \%$ for Impetigo and $85 \%$ for Melanoma. Both image based technique and questionnaire technique help to increase reliability and performance of the system.

\section{Limitations}

- This application is implemented only for three skin diseases (Eczema, Impetigo and Melanoma).

- It is implemented only for windows application so that is not yet develop for smart phones like Android, IOS etc. 
- During image acquisition, the distance between camera lens and affected skin should be $5 \mathrm{~cm}$.

- When capture the image for this application, it is mandatory to capture it without any light effects.

- It only supports English language not for other ordinary languages like Sinhala, Tamil.

\subsubsection{Online Children Skin Diseases Diagnosis System [9]}

Rule based and forward chaining inference engine methods are used to implement this model which is used to identify the skin disease. By using this system, user is allowed to identify children skin diseases via online and provide useful medical suggestions or advice timely. In this system, it consists of diagnosis module, login module, info module, report module and management module. There are two main modules called diagnosis and management module. In the diagnose module questions are asked to the user and on the basis of answers given by the user, Children's symptoms and condition are identified. This system may be an alternative for parents to identify skin diseases of children, in response to the questions about the symptoms and the condition children's skin.

\subsubsection{An automated system for recognizing disease conditions of human skin [10]}

In this model, the condition of the skin disease is identified by evaluating skin disease images by using grey normalized symmetrical simultaneous occurrence stencils (GLCM) method. The proposed system is used in an efficient and economical for the automatic recognition of skin diseases. This system is useful for the skin to reduce the error with medical diagnosis. Another is the first test for patients in rural areas, where the good doctors are missing. The system works with relational databases to the storage of implying the need for textual skin images. This system can also work for same type of images directly over feature vectors.

\subsubsection{Mobile-based Medical Assistance for \\ Diagnosing Different Types of Skin Diseases \\ Using Case-based Reasoning with Image \\ Processing [11]}

In artificial intelligence (AI), medical field is a recent area for research purpose. This paper implements a mobile based medical assistance which is used for diagnosing skin diseases by the use of CBR and image processing. This model was developed to help users to pre-examine their skin situation whether they have a disease or not. Also to increase the awareness of skin diseases on what it may do to our bodies which will lead to death or infecting other people and have a cure before it gets worse. The proposed system is successfully implemented to detect 6 different skin diseases with an accuracy of $90 \%$. The scale of symptoms, which is used for testing, is $15 \%$, for validation it is $10 \%$ and for testing it is $75 \%$. This supervised system identify diseases at the rate of $90 \%$ where the unsupervised system detect diseases at the rate of $80 \%$. The detection rate of the sample disease with the other related disease is as follows: Eczema - 88\%; Psoriasis - 61\%; Acne - 75\%; Skin Cancer - 51\%; Scabies - 43\%; and Seborrhea Dermatitis - 34\%

\subsubsection{An Innovative Skin Detection Approach Using Color based Image Retrieval Technique} [12]

The idea of "skin detection" from an image is described as the categorization of the existence pixels in that image into two skin and Non-skin classes. Many methods uses different color space to extract features for the categorization of pixels, but most of these methods does not detect different type of skin with high accuracy. The present method in this paper is implemented by using "Color based image retrieval" (CBIR) technique. In this method, first of all by finding means of CBIR method and image tiling and finding the relationship between pixel and its neighbors, a set of feature vector is prepared and then at the test stage, training is used for skin detection. Experimental results show that the proposed model identifies different type of skin with a high accuracy and it is not sensitive to illumination intensity and with the movement of face. The proposed method contains two steps such as train and test. First in training step, pure skin images were trained and then in testing steps skin area were detected from nonskin areas.

\section{Advantages:}

- Low computational complexity.

- Low time complexity.

- High accuracy for all kinds of skin.

- Capable to classify different types of skin.

Table 1: Comparison of human skin diseases detection models

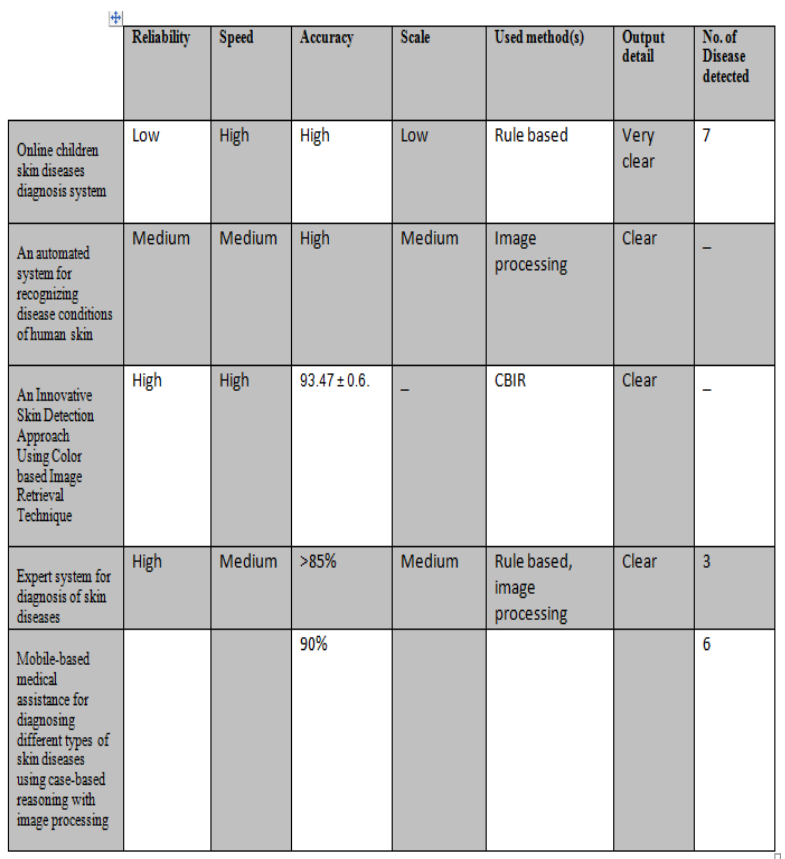

\section{CONCLUSION}

This paper gives a description of various skin diseases diagnosis models for plants, humans etc. by using image processing from which researcher can get an idea for an efficient techniques. This paper has comparison made between existing models on the basis of accuracy, speed and scale, which give a clarity output details. There are future scopes of improvements in present methodologies as no model guarantee hundred percent accuracy and is also limited to few number of skin diseases. In future present methodologies can be expanded for the detection of skin diseases in animals. A common model should be implemented for identification of all types of skin disease. 


\section{REFERENCES}

[1] Mr.Patil.S. P, Mr.Kumbhar.V.P Mr.Yadav.D.R, Ms.Ukirade.N.S Detection of Leaf Diseases by Image Processing International Journal of Advanced Research in Electronics and Communication Engineering (IJARECE) Volume 4, Issue 4, April 2015.

[2] Ken Pernezny, Monica Elliott, Aaron Palmateer and Nikolavranek Guidelines for Identification and Management of Plant Disease Problems: Part II. Diagnosing Plant Diseases Caused by Fungi, Bacteria and Viruses UF IFAS Extension.

[3] Anand.H.Kulkarni, Ashwin Patil R. K. Applying image processing technique to detect plant diseases International Journal of Modern Engineering Research (IJMER) Vol.2, Issue.5, Sep-Oct. 2012 pp-3661-3664 ISSN: 2249-6645.

[4] Shivkumar Bagde, Swaranjali Patil, Snehal Patil, Poonam Patil Artificial Neural Network Based Plant Leaf Disease Detection International Journal of Computer Science and Mobile Computing, Vol.4 Issue.4, April- 2015, pg. 900-905.

[5] Jagadeesh Devdas Pujari, Rajesh Yakkundimath and Abdulmunaf Syedhusain Byadgi Grading and Classification of Anthracnose Fungal Disease of Fruits based on Statistical Texture Features International Journal of Advanced Science and Technology Vol. 52, March, 2013

[6] Hiteshwari Sabrol, Satish Kumar Recent Studies of Image and Soft Computing Techniques for Plant Disease Recognition and Classification International Journal of Computer Applications (0975 - 8887) Volume 126 No.1, September 2015.

[7] Moureen Ahmed, Anitha Raghavendra, Dr.Mahesh Rao An Image Segmentation comparison approach for Lesion Detection and Area calculation in Mangoes International Research Journal of Engineering and Technology (IRJET) e-ISSN: 2395 -0056 Volume: 02 Issue: 05 | Aug-2015 www.irjet.net p-ISSN: 2395-0072.

[8] A.A.L.C. Amarathunga, E.P.W.C. Ellawala, G.N. Abeysekara, C. R. J. Amalraj Expert System For Diagnosis Of Skin Diseases INTERNATIONAL JOURNAL OF SCIENTIFIC \& TECHNOLOGY RESEARCH VOLUME 4, ISSUE 01, JANUARY 2015 ISSN 2277-8616.

[9] Munirah M. Yusof, Ruhaya A. Aziz, and Chew S. Fei The Development of Online Children Skin Diseases Diagnosis System International Journal of Information and Education Technology, Vol. 3, No. 2, April 2013.

[10] Anal Kumar MittraandDr. Ranjan Parekh - Automated Detection of Skin Diseases Using Texture Features.

[11] Carl Louie Aruta, Colinn Razen Calaguas, Jan Kryss Gameng, Marc Venneson Prudentino, August Anthony Chestel J. Lubaton Mobile-based Medical Assistance for Diagnosing Different Types of Skin Diseases Using Case-based Reasoning with Image Processing International Journal of Conceptions on Computing and Information Technology Vol. 3, Issue. 3, October' 2015; ISSN: 2345 - 9808 .

[12] Shervan Fekri-Ershad, Mohammad Saberi and FarshadTajeripour AN INNOVATIVE SKIN
DETECTION APPROACH USING COLOR BASED IMAGE RETRIEVAL TECHNIQUE The International Journal of Multimedia \& Its Applications (IJMA) Vol.4, No.3, June 2012.

[13] Damilola A. Okuboyejo, Oludayo O. Olugbara, and Solomon A. Odunaike (2013) - Automating Skin Disease Diagnosis Using Image Classification Proceedings of the World Congress on Engineering and Computer Science 2013, Volume II, San Francisco, USA.

[14] Damanpreet Kaur and Prabhneet Sandhu - Human Skin Texture Analysis using Image Processing Techniques International Journal of Science and Research (IJSR), India.

[15] Okuboyejo, D., Olugbara O., Odunaike S (2013). Automating Skin Disease Diagnosis Using Image Classification.

[16] Florida Extension Plant Diagnostic Network http://edis.ifas.ufl.edu/PP151.

[17] Oberti R, Marchi M, Tirelli P, Calcante A, Iriti M, Borghese A.N. 2014. Automatic detection of powdery mildew on grapevine leaves by image analysis: Optimalview-angle range to increase the sensitivity. Comput Electron Agric 104(2014), 1-8.

[18] Opstad Kruse OM, Prats-Montalbán JM, Indahl UG, Kvaal K, Ferrer A, Futsaether CM .2014. Pixel classification methods for identifying and quantifying leaf surface injury from digital images. Comput Electron Agric Vol.108(2014), 155-165. DOI:http://dx.DOI.org/10.1016/j.compag.2014.07.010.

[19] Tejal Deshpande, Sharmila Sengupta, and K.S.Raghuvanshi, "Grading \& Identification of Disease in Pomegranate Leaf and Fruit," IJCSIT, vol. 5 (3), pp 4638-4645, 2014

[20] P.Revathi and M.Hemalatha, "Classification of Cotton Leaf Spot Diseases Using Image Processing Edge Detection Techniques," IEEE International Conference on Emerging Trends in Science, Engineering and Technology (INCOSET), Tiruchirappalli, pp 169-173, 2012.

[21] Ms. Kiran R. Gavhale, Prof. Ujwalla Gawande, and Mr. Kamal O. Hajari, "Unhealthy Region of Citrus Leaf Detection using Image Processing Techniques," IEEE International Conference on Convergence of Technology (I2CT), Pune, pp 1-6, 2014.

[22] Monika Jhuria, Ashwani Kumar and Rushik esh Borse, "Image processing for smart farming detection of disease and fruit grading," IEEE 2nd International Conference on Image Information Processing (ICIIP), Shimla, pp 521-526, 2013.

[23] Ganesan P, Priya Chakravarty, Shweta Verma Segmentation Of Natural Color Images In Hsi Color Space Based On Fcm Clustering. International Journal Of Advanced Research In Computer Engineering \& Technology (Ijarcet) Volume 3 Issue 3, March 2014.

[24] Shiv Ram Dubey, Pushkar Dixit, Nishant Singh , Jay Prakash Gupta . Infected Fruit Part Detection Using KMeans Clustering Segmentation Technique International Journal Of Artificial Intelligence And Interactive Multimedia, Vol. 2, $\mathrm{N}^{\mathrm{o}} 2$. 
[25] O'Malley, J., and Ginsburg, R. (2010). Discriminationon the Basis of Color. http://www.lexology.com/library/detail.aspx?g=9013b94 9- $1 \mathrm{f} 70$ -

[26] k-means clustering Available at: http://www.mathworks.com/help/stats/kmeans.html.(Acc essed on 25April 2015)

[27] (Accessed on 22 May 2015) Blast (leaf and collar) IRRI Rice Knowledge Bank, Available at: http://www.knowledgebank.irri.org/training/factsheets/pe stma nagement/diseases/item/blast-leaf-collar. (Accessed on 25 April 2015).

[28] bwarea, Area of objects in binary image, Available at: http://www.mathworks.com/help/images/ref/bwarea.html (Accessed on 22 May 2015)

[29] H. Al-Hiary, S. Bani-Ahmad, M. Reyalat, M. Braik and Z. ALRahamneh, Fast And Accurate Detection and Classification of Plant diseases, International Journal of Computer Applications (0975 -8887),Volume 17-No.1, March 2011

[30] Nadia Smaoui, Souhir Bessassi," A developed system for melanoma diagnosis",2013, International Journal of
Computer Vision and Signal Processing, 3(1), 1017(2013)

[31] Teresa Mendonca, Pedro M. Ferreira,Jorge S. Marques, Andre R. S. Marcal, Jorge Rozeira," PH2 - A dermoscopic image database for research and benchmarking",2013, 35th Annual InternationalConference of the IEEE EMBS Osaka, Japan, 3 - 7 July, 2013.

[32] Kawsar Ahmed, Tasnuba Jesmin, Md. Zamilur Rahman," Early Prevention and Detection of Skin Cancer Risk using Data Mining",2013, InternationalJournal of Computer Applications (0975 - 8887) Volume 62- No.4, January 2013

[33] Mariam A.Sheha, Mai S.Mabrouk," Automatic Detection of Melanoma Skin Cancer",[2012], International Journal of Computer Applications (0975- 8887)March,2012.

[34] Zulkifli Bin Husain, Abdul Hollis Bin Abdul Aziz, Ali Yean Bin MdShakaff, RohaniBinti S Mohamed Farook, "Feasibility Study on Plant Chili Disease Detection Using Image Processing Techniques" in Proceedings of IEEE International Conference on Intelligent Systems Modelling and Simulation, PP : 291-296,2012. 